\title{
Association between PNPLA3 rs738409 polymorphism and decreased \\ kidney function in postmenopausal type 2 diabetic women with or without non-alcoholic fatty liver disease
}

Running title: PNPLA3 and risk of CKD

Alessandro Mantovani, $\mathrm{MD}^{1}$, Chiara Zusi, $\mathrm{PhD}^{1,2}$, Elena Sani, $\mathrm{MD}^{1}$, Antonio Colecchia, $\mathrm{MD}^{3}$, Giuseppe Lippi, MD ${ }^{4}$, Gianluigi Zaza, $M D^{5}$, Luca Valenti, MD ${ }^{6}$, Christopher D. Byrne, MB BCh, $\mathrm{PhD}^{7,8}$, Claudio Maffeis, $\mathrm{MD}^{2}$, Enzo Bonora, $\mathrm{MD}^{1}$, Giovanni Targher, $\mathrm{MD}^{1}$

${ }^{1}$ Division of Endocrinology, Diabetes and Metabolism, Department of Medicine, University Hospital of Verona, Verona, Italy

${ }^{2}$ Pediatric Diabetes and Metabolic Disorders Unit, Department of Surgical Sciences, Dentistry, and Pediatrics, and Gynaecology, University Hospital of Verona, Verona, Italy

${ }^{3}$ Gastroenterology Unit, Azienda Ospedaliera Universitaria Integrata of Verona, Verona, Italy

${ }^{4}$ Section of Clinical Biochemistry, University and Azienda Ospedaliera Universitaria Integrata of Verona, Verona, Italy

${ }^{5}$ Renal Unit, Department of Medicine, University and Azienda Ospedaliera Universitaria Integrata of Verona, Verona, Italy

${ }^{6}$ Department of Pathophysiology and Transplantation, University of Milan, and Translational Medicine - Department of Transfusion Medicine and Hematology, Fondazione IRCCS Ca' Granda Ospedale Maggiore Policlinico Milano, Milan, Italy

${ }^{7}$ Nutrition and Metabolism, Faculty of Medicine, University of Southampton, UK

${ }^{8}$ Southampton National Institute for Health Research Biomedical Research Centre, University Hospital Southampton, Southampton General Hospital, Southampton SO16 6YD, UK

Word count: abstract 250; text 3,839 (excluding title page, abbreviation list, abstract, references, figure legends and tables); n. 3 Tables; n. 1 Figure; n. 3 supplementary Tables

Address of correspondence:

Prof. Giovanni Targher, MD

Section of Endocrinology, Diabetes and Metabolism

University and Azienda Ospedaliera Universitaria Integrata

Piazzale Stefani, 1

37126 Verona, Italy

Phone: $+39 / 045-8123748$

Fax: $+39 / 045-8027314$

E-mail: giovanni.targher@univr.it 


\section{LIST OF ABBREVIATIONS}

$A C R$, albumin-to-creatinine ratio

$\mathrm{ALT}$, alanine aminotransferase

AST, aspartate aminotransferase

$\mathrm{BMI}$, body mass index

$\mathrm{Cl}$, confidence interval

CKD, chronic kidney disease

CKD-EPI, Chronic Kidney Disease Epidemiology Collaboration

CRP, C-reactive protein

eGFR, estimated glomerular filtration rate

FIB-4, fibrosis 4

FLI, fatty liver index

GGT, gamma-glutamyltransferase

HOMA-IR, Homeostasis model assessment-insulin resistance

MDRD, Modification of Diet in Renal Disease

NAFLD, nonalcoholic fatty liver disease

$\mathrm{NASH}$, nonalcoholic steatohepatitis

OR, odds ratio

PNPLA3, patatin-like phospholipase domain-containing protein 3

SNP, single nucleotide polymorphism

T2DM, type 2 diabetes mellitus 


\section{ABSTRACT}

Aim: Evidence is emerging that PNPLA3 rs738409 polymorphism (the major genetic variant associated with susceptibility to non-alcoholic fatty liver disease [NAFLD]) is associated with chronic kidney disease (CKD) in nondiabetic individuals. Currently, little is known about this association in type 2 diabetic (T2DM) patients with and without NAFLD.

Methods: We studied 101 Caucasian post-menopausal women with T2DM, consecutively attending our diabetes outpatient service during a 3-month period. Glomerular filtration rate

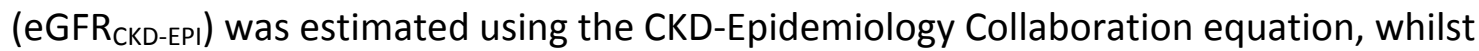
albuminuria was measured with an immunonephelometric assay on morning spot urine samples. NAFLD was detected either by fatty liver index (FLI $\geq 60, n=101)$ or by ultrasonography $(n=77)$. Genotyping was performed by TaqMan-Based RT-PCR system.

Results: Eight patients had G/G, 41 G/C and 52 C/C PNPLA3 rs738409 genotypes, and 21 (20.8\%) patients had CKD (eGFR CKD-EPI $_{60} \mathrm{ml} / \mathrm{min} / 1.73 \mathrm{~m}^{2}$ or abnormal albuminuria). Compared to those with $\mathrm{G} / \mathrm{C}$ or $\mathrm{C} / \mathrm{C}$ genotypes, patients with $\mathrm{G} / \mathrm{G}$ genotype had significantly lower eGFR $\mathrm{RKD}_{\mathrm{CEPI}}$ (63.7 \pm 11 vs. $77.4 \pm 17$ vs. $\left.81.9 \pm 15 \mathrm{ml} / \mathrm{min} / 1.73 \mathrm{~m}^{2}, p=0.014\right)$ and higher prevalence of CKD (50\% vs. $24.4 \%$ vs. $13.5 \%, p=0.04)$. After adjustment for age, diabetes duration, hemoglobin A1c, HOMA-estimated insulin resistance, systolic blood pressure, hypertension treatment and FLI $\geq 60$, rs738409 G/G genotype was independently associated with lower eGFR $R_{\text {CKD-EPI }}(\beta$ coefficient: 15.5, 95\% $\mathrm{Cl}-26.0$ to $-5.0, p=0.004$ ) and higher risk of CKD (adjusted-odds ratio 8.05, 95\%Cl 1.26-41.4, $p=0.03)$.

Conclusion: Regardless of the presence of NAFLD, in post-menopausal women with T2DM, the G/G genotype of rs738409 in the PNPLA3 gene was independently associated with lower eGFR $R_{\text {CKD-EPI }}$ and higher prevalence of CKD.

Keywords: adiponutrin; CKD; chronic kidney disease; NAFLD; type 2 diabetes 


\section{INTRODUCTION}

Non-alcoholic fatty liver disease (NAFLD) has become the most common cause of chronic liver disease in many parts of the world, affecting up to $30 \%$ of adults in high-income countries (1). The prevalence of NAFLD is much higher in people with type 2 diabetes mellitus (T2DM) (occurring in up to $\sim 70 \%$ of these patients), who are a group of individuals at high-risk of developing the more severe histologic forms of NAFLD, such as non-alcoholic steatohepatitis (NASH), advanced fibrosis and cirrhosis (2). It is now becoming increasingly clear that the clinical and economic burden of NAFLD does not only affect the risk of liver-related complications, but also affects the risk of developing both cardiovascular disease and other extra-hepatic diseases that have a significant impact on healthcare expenditure $(1,2)$. Recently, a number of studies have repeatedly shown that NAFLD is associated with an increased risk of both prevalent and incident chronic kidney disease (CKD) in patients with and without T2DM $(3,4)$, independent of common cardio-renal risk factors. Identification of novel risk factors for CKD is clinically relevant, as CKD is a common pathologic condition worldwide that is associated with high morbidity, disability, mortality and healthcare costs (5-7).

Among the genetic factors that may influence the development and progression of NAFLD, the minor allele G of rs738409, i.e., a non-synonymous single nucleotide polymorphism (SNP) in the patatin-like phospholipase domain-containing protein 3 (PNPLA3) gene encoding an lle148Met change, has been recognized to be the major common genetic variant associated with susceptibility to NASH $(8,9)$. The PNPLA3 gene is mostly expressed in the liver and has an acyl hydrolase activity $(8,9)$. In particular, the $\mathrm{G}$ allele of $\mathrm{rs} 738409$ is associated with the loss of hydrolyzing function of the protein, thus resulting in accumulation of lipid droplets into the hepatocytes $(8,9)$. Recently, some studies suggested that the presence of the $G$ allele of 
rs738409 is associated with lower estimated glomerular filtration rate (eGFR) and higher prevalence of CKD in nondiabetic individuals, irrespective of the coexistence of NAFLD $(10,11)$.

To our knowledge, it is currently uncertain whether such an association also occurs in patients with T2DM (i.e., a group of individuals at high-risk of developing both CKD and NAFLD). Therefore, the major aim of our cross-sectional study was to examine whether, and to what extent, PNPLA3 rs738409 polymorphism (the major genetic variant associated with susceptibility to NASH) was associated with decreased kidney function in a sample of adult patients with T2DM (both with and without coexisting NAFLD).

\section{METHODS}

Patients

In this exploratory analysis, we studied 101 post-menopausal Caucasian women with non-insulin treated T2DM, who consecutively attended our diabetes outpatient service during a 3-month period (from October to December 2017). We excluded all patients with: (a) history of significant alcohol consumption (i.e., >20 grams of alcohol per day) and other known causes of chronic liver diseases (e.g., virus, drugs, autoimmunity and hemochromatosis); (b) history of overt dysthyroidism, cirrhosis of any etiology, cancer and end-stage renal disease (defined as eGFR <15 $\mathrm{ml} / \mathrm{min} / 1.73 \mathrm{~m}^{2}$ or chronic dialysis); and (c) treatment with hormone replacement therapy, steroids or anti-osteoporotic agents. In this analysis, we included only post-menopausal women, because this study was primarily designed for exploring the association between NAFLD, bone mineral density and circulating levels of bone turnover biomarkers in post-menopausal women with non-insulin treated T2DM (12). 
The local Ethics Committee approved the study protocol. All participants gave their written informed consent for participation in this research.

\section{Clinical and laboratory data}

Body mass index (BMI) was measured as kilograms divided by the square of height in meters.

Waist circumference was measured at the midpoint between the lowest rib and the iliac crest. Blood pressure was measured with a standard sphygmomanometer after the patient had been seated quietly for at least 5 minutes. Patients were considered to have hypertension if their blood pressure was $\geq 140 / 90 \mathrm{mmHg}$ or if they were taking any anti-hypertensive drug. Information on the type of menopause (physiological or surgical), smoking and use of medications was obtained from all patients via interviews during medical examinations.

Venous blood samples were collected in the morning after an overnight fast. Measurements of serum glucose, lipids, creatinine (measured using a Jaffé rate blanked and compensated assay), aspartate aminotransferase (AST), alanine aminotransferase (ALT), gamma-glutamyltransferase (GGT), high-sensitivity $\mathrm{C}$ reactive protein (hs-CRP) and other biochemical blood parameters were obtained using standard laboratory procedures at the central Laboratory of our hospital. Hemoglobin A1c (HbA1c) was measured using the high-performance liquid chromatography analyzer Tosoh-G7 (Tosoh Bioscience Inc., Tokyo, Japan). Fasting insulin levels were measured using a chemiluminescent immunoassay (LIAISON, DiaSorin, Saluggia, Italy). Homeostasis model assessment (HOMA-IR) score was used for estimating insulin resistance. 
GFR was estimated using both the Chronic Kidney Disease Epidemiology Collaboration (CKD-EPI) equation (13) and the four-variable Modification of Diet in Renal Disease (MDRD) study equation (14). However, the CKD-EPI equation, which uses the same four variables as the MDRD study equation, has been shown to be more accurate than the MDRD study equation for estimating GFR in different populations, as well as for predicting the risk of mortality in patients with T2DM (15). Urinary albumin excretion was assessed with an immunonephelometric assay (BeckmanCoulter IMMAGE; Beckman-Coulter Instruments, Fullerton, CA, USA) on a morning spot urine sample and expressed as the albumin-to-creatinine ratio (ACR); abnormal albuminuria was defined as a urinary $A C R \geq 30 \mathrm{mg} / \mathrm{g}$ creatinine.

In this study, chronic kidney disease (CKD) was defined as the presence of eGFR $\mathrm{CKD}_{\mathrm{CPI}}<60$ $\mathrm{mL} / \mathrm{min} / 1.73 \mathrm{~m}^{2}$ or abnormal albuminuria. Pre-existing history of ischemic heart disease was defined as a documented history of myocardial infarction, angina pectoris or coronary revascularization procedures. Presence of diabetic retinopathy (diagnosed with fundoscopy after pupillary dilation) was also recorded.

Definition of NAFLD

In this study, we used two different methods for diagnosing NAFLD: the fatty liver index (FLI, which was available in the whole sample, $n=101$ ) and liver ultrasonography (available only in a subgroup of 77 subjects) after excluding individuals with other causes of liver diseases (as described in the exclusion criteria). In particular, we calculated the fatty liver index (FLI), i.e., a widely used marker of NAFLD (16), for identifying patients with NAFLD. In accordance with previous reports (17-19), a FLI $\geq 60$ indicates individuals with hepatic steatosis on ultrasound. FLI was calculated as follows (19): $\mathrm{FLI}=\left[\mathrm{e}^{0.953} \times \mathrm{Ln}\right.$ (triglycerides) $+0.139 \times \mathrm{BMI}+0.718 \times \mathrm{Ln}(\mathrm{GGT})+$ 
$0.053 \times$ waist circumference -15.745$)] /\left[1+\mathrm{e}^{0.953} \times \operatorname{Ln}(\right.$ triglycerides $)+0.139 \times \mathrm{BMI}+0.718 \times$ Ln(gamma-glutamyltransferase) $+0.053 \times$ waist circumference -15.745$] \times 100$. A liver ultrasonography was also conducted on a subgroup of patients ( $n=77 ; 76.2 \%$ of total) by a single expert physician (using an Esaote MyLab 70 ultrasound with a $4 \mathrm{MHz}$ probe), who was blinded to the laboratory findings of participants. Hepatic steatosis was diagnosed according to specific ultrasonographic characteristics, such as diffuse hyper-echogenicity of the liver relative to kidneys, ultrasonographic beam attenuation, and poor visualization of intra-hepatic vessel borders and diaphragm $(16,20)$. Therefore, NAFLD was defined either by FLI $\geq 60$ or by presence of hepatic steatosis on ultrasonography, separately, after exclusion of significant alcohol intake or other identifiable causes of liver disease. No large studies have validated the use of FLI in predicting hepatic fat content on imaging techniques in patients with T2DM, although the few published studies (performed in small series of patients) provided inconclusive results $(21,22)$. In our study, the Cohen's kappa coefficient for agreement between FLI $\geq 60$ and ultrasonography for detecting NAFLD was relatively low $(k=0.22)$. Finally, we also used a validated cutoff of fibrosis-4 score (FIB-4 >2.67) for non-invasively diagnosing the presence of advanced NAFLD fibrosis among individuals aged $\geq 65$ years $(16,23)$.

\section{Genetic analysis}

Genomic DNA was extracted from peripheral blood leukocytes using QIAamp DNA Blood Mini Kit (Qiagen, Germany). Genotyping of rs738409 in PNPLA3 gene was carried out by a predesigned TaqMan probe (Applied Biosystem, California, USA), underling the I148M substitution, according to manufacturers' protocol. Polymorphism genotyping was performed using 7900 HT Real Time PCR (Applied Biosystem, California, USA). Distribution of the genotype was in Hardy-Weinberg 
equilibrium and the call rate exceeded $99 \%$. The allele G of rs738409 variant was present in $28.2 \%$ of chromosomes.

\section{Statistical Analysis}

Due to the exploratory (hypothesis-generating) design of the study, it was not possible to compute a sample size. Data are expressed as means $\pm S D$, medians (inter-quartile ranges, IQR) or percentages. The PNPLA3 rs738409 associations were assessed using an additive genetic model. The Fisher's exact test for categorical variables, the one-way ANOVA for normally distributed continuous variables and the Kruskal-Wallis test for non-normally distributed variables were used to test the differences in clinical and biochemical characteristics among patients stratified by PNPLA3 rs738409 polymorphism (Table 1). The association between PNPLA3 rs738409 variant and values of both eGFR $\mathrm{CKD}_{\text {EPI }}$ and eGFR $\mathrm{MDRD}_{\text {(included as continuous }}$ measures, i.e. for each SD decrement) was tested using both an unadjusted linear regression model and the following two multivariable regression models: (i) model 1 adjusted for age, duration of diabetes, HbA1c, HOMA-IR score, systolic blood pressure, use of any antihypertensive drug (i.e., beta-blockers, renin-angiotensin system inhibitors, calcium-channel antagonists or diuretics) and presence of NAFLD (as detected by FLI $\geq 60$ ), and (ii) model 2 further adjusted for smoking history, statin use, pre-existing ischemic heart disease and abnormal albuminuria (i.e., urinary $A C R \geq 30 \mathrm{mg} / \mathrm{g}$ ) (Table 2). Moreover, we also tested the association between PNPLA3 rs738409 variant and the risk of prevalent CKD (defined as eGFR $\mathrm{RKD}_{\mathrm{CPI}}<60$ $\mathrm{ml} / \mathrm{min} / 1.73 \mathrm{~m}^{2}$ or abnormal albuminuria) by using both an unadjusted logistic regression model and an adjusted logistic regression model 1 , including the same list of the aforementioned covariates (Table 3). In these multivariable regression models we did not additionally adjust for anthropometric parameters and serum triglyceride and liver enzyme levels, because these 
variables are already included in the FLI equation. In the subgroup of patients with available liver ultrasound data $(n=77)$, we also repeated the same multivariable linear and logistic regression models, including the presence of NAFLD (diagnosed with ultrasonography, instead of FLI $\geq 60$ ) among the covariates (Supplementary Tables 1 and 2). Covariates included in all multivariable regression models were selected as potential confounding factors based on their biological plausibility. A $p$-value $<0.05$ was considered statistically significant. Statistical analyses were performed using STATA software, version 14.2 (STATA, College Station, Texas, USA).

\section{RESULTS}

Among the 101 post-menopausal elderly women with T2DM included in the study (mean \pm SD: age $71.7 \pm 9$ years; BMI $\left.29.5 \pm 5 \mathrm{~kg} / \mathrm{m}^{2} ; \mathrm{HbA} 1 \mathrm{c} 51 \pm 8 \mathrm{mmol} / \mathrm{mol}\right), 8$ (7.9\%) women had $\mathrm{G} / \mathrm{G}, 41$ (40.6\%) had G/C, and 52 (48.5\%) had C/C PNPLA3 rs738409 genotype, respectively. In addition, $21\left(20.8 \%\right.$ ) of these women had CKD (defined as eGFR $\mathrm{CKD}_{\text {EPI }}<60 \mathrm{ml} / \mathrm{min} / 1.73 \mathrm{~m}^{2}$ or abnormal albuminuria). In the subgroup of 77 patients in whom data on liver ultrasonography were also available, 62 (80.5\%) patients met the diagnostic criteria for NAFLD. Among those with NAFLD, 36 patients had mild hepatic steatosis and 26 had moderate-to-severe steatosis on ultrasonography, respectively.

Table 1 shows the clinical and biochemical characteristics of participants, stratified by PNPLA3 rs738409 polymorphism. Compared to patients with either $\mathrm{G} / \mathrm{C}$ or $\mathrm{C} / \mathrm{C}$ genotypes, those with G/G genotype had a significantly higher prevalence of CKD ( $50 \%$ vs. $24.4 \%$ vs. $13.5 \%$ ) and also tended to have higher abnormal albuminuria. Age, BMI, waist circumference, duration of diabetes, smoking, blood pressure, complete blood count, HbA1c, HOMA-IR score, plasma lipid 
profile, hs-CRP, albumin, liver enzymes, diabetic retinopathy, ischemic heart disease, as well as the use of oral hypoglycemic agents, statins and anti-hypertensive drugs did not significantly differ among the three groups of patients. Additionally, the prevalence of hepatic steatosis (as detected either by FLI $\geq 60$ or by ultrasonography) and advanced NAFLD fibrosis (as defined by FIB-4 score >2.67) was comparable among the three patient groups.

Notably, as shown in Figure 1, the values of both eGFR $\mathrm{CKD}_{\text {EPI }}$ and eGFR $\mathrm{CKD}_{\text {EPI }}$ decreased progressively in relation to the PNPLA3 rs 738409 variant $(p=0.014$ and $p=0.025$ for the trend by one-way ANOVA, respectively).

Supplementary Table 1 shows the main clinical/biochemical data and PNPLA3 rs738409 genotype of patients, stratified by both CKD and NAFLD (as detected by FLI $\geq 60$ ). The four subgroups of patients significantly differed in terms of BMI, waist circumference, HOMAinsulin resistance, plasma lipid profile and GGT concentrations. Conversely, the percentage of individuals with the risk allele (G) of rs738409 was not significantly different among the patient groups.

As reported in Table 2, the risk allele (G) of rs738409 was significantly associated with lower eGFR $_{\text {CKD-EPI }}(\beta$ coefficient $\mathbf{- 1 5 . 2 , 9 5 \% C l ~ - 2 5 . 4 ~ t o ~}-\mathbf{5 . 1}, \boldsymbol{p}=\mathbf{0 . 0 0 4})$, even after adjustment for age, diabetes duration, HbA1c, HOMA-IR score, systolic blood pressure, hypertension treatment and NAFLD (as assessed by FLI) (model 1). After further adjustment for smoking, statin use, history of ischemic heart disease and abnormal albuminuria (model 2), the results remained essentially unchanged, showing that the risk allele (G) of rs738409 was independently associated with lower eGFR $_{\text {CKD-EPI }}(\beta$ coefficient $\mathbf{- 1 5 . 5 , 9 5 \% C l ~} \mathbf{- 2 6 . 0}$ to $\mathbf{- 5 . 0}, \boldsymbol{p}=\mathbf{0 . 0 0 4})$. Other variables that were 
independently associated with lower eGFR ${ }_{C K D-E P I}$ were older age $(\beta$ coefficient $-\mathbf{0 . 7 4 , 9 5 \% C l}-\mathbf{1 . 1}$ to $-0.4, p<0.001)$ and presence of NAFLD ( $\beta$ coefficient $-9.50,95 \% \mathrm{Cl}-15.7$ to $-3.3, p=0.003)$. As also shown in the table, very similar results were found even when we used the MDRD study equation (instead of the CKD-EPI equation) for estimating GFR.

Table 3 shows the association between the risk allele (G) of PNPLA3 rs738409 and the presence of CKD (defined as eGFR $\mathrm{CKD}_{\text {-EPI }}<60 \mathrm{ml} / \mathrm{min} / 1.73 \mathrm{~m}^{2}$ or abnormal albuminuria). In univariable logistic regression analysis (unadjusted model), the risk allele (G) of rs738409 was significantly associated with a 6.5-fold increased risk of prevalent CKD. Adjustment for age, diabetes duration, HbA1c, HOMA-IR score, systolic blood pressure, hypertension treatment and presence of NAFLD (as assessed by FLI) did not substantially modify these results.

Interestingly, when we restricted the aforementioned multivariable linear and logistic regression analyses to the subgroup of patients $(n=77)$ with available liver ultrasound data (Supplementary Table 1 and Supplementary Table 2), the presence of risk allele (G) of rs738409 in PNPLA3 gene was confirmed to be independently associated with lower eGFR $\mathrm{CKD}_{\text {-EPI }}$ (or lower eGFR $\mathrm{R}_{\mathrm{MDRD}}$ ) and with higher prevalence of CKD, even after adjusting for NAFLD (as detected by ultrasonography) and other potential confounding factors.

\section{DISCUSSION}

This is the first cross-sectional study aimed at examining the association of PNPLA3 rs738409 polymorphism with kidney function and risk of prevalent CKD (defined as eGFR $R_{C K D-E P I}<60$ 
$\mathrm{ml} / \mathrm{min} / 1.73 \mathrm{~m}^{2}$ or abnormal albuminuria) in patients with T2DM, a group of individuals at high risk for developing both CKD and NAFLD.

The main and novel findings of our study were that the presence of the risk allele (G) of rs738409 was significantly associated with lower values of both eGFR $R_{C K D-E P I}$ and EGFR $R_{M D R D}$, as well as with a greater risk of CKD (defined as eGFR CKD-EPI $<60 \mathrm{ml} / \mathrm{min} / 1.73 \mathrm{~m}^{2}$ or abnormal albuminuria) in an outpatient sample of Caucasian post-menopausal women with T2DM. Notably, these associations were independent of age, duration of diabetes, HbA1c, HOMA-IR score, smoking, systolic blood pressure, hypertension treatment, statin use, prior history of ischemic heart disease and NAFLD (as detected either by FLI or by ultrasonography). In addition, other risk factors that were independently associated with reduced kidney function were older age and coexisting NAFLD.

This latter finding is supported by the results of multiple observational studies showing that the presence and severity of NAFLD is associated with an increased risk of both prevalent and incident CKD in patients with and without T2DM $(3,4)$. Unfortunately, we did not assess hepatic steatosis by ultrasound in all subjects and we did not attempt to grade accurately the severity of hepatic steatosis in those subjects in whom ultrasound was undertaken. That said, in the subgroup of $\mathbf{7 7}$ patients with available liver ultrasound data we failed to find an association between eGFR values and ultrasonographic confirmed hepatic steatosis. Therefore, we did not examine the association between hepatic fat content and eGFR or albuminuria according to PNPLA3 rs738409 genotype. However, in line with previous studies showing that hepatic fibrosis stage is the strongest predictor of all-cause death and cardiovascular events in individuals with NAFLD $(1,2)$, it is reasonable to assume that patients with NASH and hepatic 
fibrosis are at substantially higher risk of incident CKD than those patients with simple steatosis alone $(3,4)$, since it is likely that the mechanisms linking hepatic fibrosis and increased cardiovascular events are similar to those linking hepatic fibrosis and CKD.

Our findings corroborate and expand the results of two recent studies, showing that PNPLA3 rs738409 polymorphism was significantly associated with an increased risk of prevalent CKD in predominantly non-obese, nondiabetic men and women $(10,11)$. In a preliminary cross-sectional study of nearly 200 non-obese, nondiabetic middle-aged individuals (about a third of whom had biopsy-proven non-cirrhotic NAFLD), Musso et al. reported that the G allele of rs738409 was significantly associated with lower eGFR $\mathrm{CKD}_{\text {-EPI }}$ values and with a higher prevalence of CKD both in patients with and in those without NAFLD (10). However, it was unclear from this preliminary study (published as a letter) whether these associations were also adjusted for established cardio-renal risk factors. Also, in an exploratory cross-sectional and longitudinal study, Oniki et al. (11) examined the relationship between PNPLA3 genotype and kidney function decline in 740 Japanese elderly men and women ( $10 \%$ with established diabetes) recruited during a healthscreening program. These authors showed that carriers of the PNPLA3 G/G genotype had lower

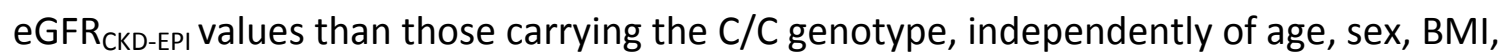
diabetes, hypertension, dyslipidemia and ultrasound-defined NAFLD. Notably, in a subgroup of nearly 350 non-obese men and women followed for a median period of 5 years, the same authors also confirmed that the PNPLA3 G/G genotype was independently associated with a significant decline in eGFR values (11). However, the generalizability of the findings of this study to other ethnic groups remains untested. 
That said, we believe that the evidence from this and the two aforementioned studies is of potential clinical importance for management of both patients with and without T2DM. The combined data support the assertion that PNPLA3 genotyping might be useful for identifying individuals who are more at risk of developing severe liver disease in NAFLD. This may be particularly important for individuals without established diabetes, since diabetes is a recognized factor that is causally implicated in the development of more severe liver disease in NAFLD. Additionally, PNPLA3 genotyping might also be useful for identifying those subjects with a higher risk of developing NAFLD-related extra-hepatic complications, such as CKD, through the promotion of specific prevention and treatment strategies for carriers of the PNPLA3 rs738409 G/G genotype.

It is known that the PNPLA3 gene encodes a trans-membrane polypeptide chain exhibiting triglyceride hydrolase activity, which is highly expressed on endoplasmic reticulum and lipid membranes of hepatocytes and adipocytes (24-26). The rs738409 C>G SNP in the PNPLA3 gene, encoding the isoleucine to methionine variant at protein position 148 (I148M), impairs the phospholipase activity of the enzyme, and accumulates at the surface of lipid droplets, where it acquires the ability to alter triglyceride and phospholipid turnover, thus inducing hepatic fat accumulation (24-26). To date, while the role of PNPLA3 rs738409 gene variant in the development and progression of NAFLD has been established $(8,9,27)$, the putative biological mechanisms underlying the association between the $\mathrm{G}$ allele of $\mathrm{rs} 738409$ and risk of CKD remain poorly known. As NAFLD is closely associated with an increased risk of CKD in both patients with and without T2DM $(3,4)$, the most obvious explanation for our findings is that the association between the G allele of rs738409 and kidney dysfunction is simply an epiphenomenon of coexisting NAFLD. However, it should be noted that in our study the significant associations of 
the $\mathrm{G}$ allele of rs738409 with both lower eGFR and higher prevalence of CKD persisted even after adjustment for NAFLD (detected by either FLI or ultrasonography) and other established cardiorenal risk factors (such as age, diabetes duration, HbA1c, HOMA-IR score, smoking, hypertension, statin use, prior ischemic heart disease and abnormal albuminuria). Experimentally, there is evidence showing that PNPLA3 is also expressed in sinusoidal pericytes (being this evidence stronger for hepatic stellate cells) that store retinol and regulate its release in response to multiple metabolic signals (28), and carriage of the PNPLA3 risk variant is associated with an increased release of proinflammatory and profibrogenic factors (29). In kidney, pericytes have also become an intensively studied cell population in renal biology and pathophysiology (30). Renal pericytes are stromal cells that have been reported to play critical roles in angiogenesis, regulation of renal medullary and cortical blood flow, and may also serve as progenitors of interstitial myofibroblasts in kidney fibrogenesis (30). Although it remains unclear whether PNPLA3 may also be involved in the regulation of functions of renal pericytes, it is plausible to hypothesize that the 148M PNPLA3 risk allele (impairing the phospholipase activity of the enzyme) might favor an unbalanced activation of pericytes in the kidneys, thus promoting the development of liver and kidney fibrosis (31). Indeed, kidney fibrosis is considered as the underlying pathological process of CKD. It is a complex and progressive process, in which a number of different acquired and genetic factors and molecular mediators (including also various phospholipases, such as PLA2 and PLD4) are involved (30,32). However, further mechanistic studies are needed to better elucidate the role of the I148M PNPLA3 variant on the development of kidney fibrosis.

Our study has some important limitations that should be mentioned. First, the cross-sectional design of the study limits our ability to establish the causality and temporality of the observed 
associations, although this limitation is mitigated by the fact that the genetic variants under study are inherited and, therefore, reverse causation does not apply. Second, it is possible that there may be a selection bias from including an outpatient sample of Italian post-menopausal women with non-insulin treated T2DM. Hence, our results might not be necessarily generalizable to other ethnic groups or to other patients with T2DM, not attending a diabetes outpatient service. Third, we did not perform either a liver biopsy, which is the reference method for diagnosing and staging NAFLD, or a magnetic resonance-proton density fat fraction imaging, which is an accurate method for measuring hepatic fat content (16). However, we believe that it would have been hazardous to perform liver biopsies in these T2DM patients with fairly normal serum liver enzymes. Finally, the number of individuals with isolated abnormal albuminuria was relatively low, thus limiting our ability to examine the differential impact of PNPLA3 rs738409 polymorphism on eGFR and albuminuria, separately.

Notwithstanding these limitations, our study has some important strengths, including the consecutive enrollment of the study population, the completeness of the database, the adjustment for multiple common risk factors for CKD, and the exclusion of patients with important comorbidities (e.g., advanced kidney disease, cirrhosis or cancer). We believe that including patients with these morbid conditions might have confounded the interpretation of data.

In conclusion, the results of this cross-sectional study suggest that the $\mathrm{G}$ allele of rs738409 is associated with decreasing kidney function and CKD (defined as eGFR CKD-EPI $_{1}<60 \mathrm{ml} / \mathrm{min} / 1.73 \mathrm{~m}^{2}$ or abnormal albuminuria) in post-menopausal women with T2DM, independent of the presence of NAFLD and other established cardio-renal risk factors. Future larger follow-up and mechanistic 
studies are certainly needed to better understand the link between PNPLA3 rs738409 polymorphism and risk of decreased kidney function and a possible interaction with hepatic fat content.

Disclosure Statement: The Authors have no potential conflicts of interest to disclose.

Funding Sources: GT is supported in part by grants from the University School of Medicine of Verona, Verona, Italy. CDB is supported in part by grants from the Southampton National Institute for Health Research Biomedical Research Centre. LV is supported by myFIRST AIRC grant n.16888 for the EPIDEMIC-NAFLD project, Ricerca Finalizzata 2016 Ministero della Salute - RF2016-02364358.

Authors' Contributions: AM and GT conceived and designed the study. CZ performed genotyping. AC performed liver ultrasonography. AM, ES and GL researched data and reviewed/edited the manuscript. $A C, G Z, G L, L V, C D B, C M$ and EB contributed to discussion and reviewed/edited the manuscript. AM and GT analyzed the data and wrote the manuscript. AM and GT are the guarantors of this work and, as such, had full access to all the data of the study and take responsibility for the integrity and accuracy of data. All authors approved the final version of the manuscript.

\section{REFERENCES}

1. Younossi Z, Anstee QM, Marietti M, Hardy T, Henry L, Eslam M, George J, Bugianesi E. Global burden of NAFLD and NASH: trends, predictions, risk factors and prevention. Nat Rev Gastroenterol Hepatol 2018; 15: 11-20. 
2. Targher G, Lonardo A, Byrne CD. Nonalcoholic fatty liver disease and chronic vascular complications of diabetes mellitus. Nat Rev Endocrinol 2018; 14: 99-114.

3. Musso G, Gambino R, Tabibian JH, Ekstedt M, Kechagias S, Hamaguchi M, Hultcrantz R, Hagström H, Yoon SK, Charatcharoenwitthaya P, George J, Barrera F, Hafliđadóttir S, Björnsson ES, Armstrong MJ, Hopkins L, Gao X, Francque S, Verrijken A, Yilmaz Y, Lindor KD, Charlton M, Haring R, Lerch MM, Rettig R, Völzke H, Ryu S, Li G, Wong LL, Machado M, Cortez-Pinto H, Yasui K, Cassader M. Association of non-alcoholic fatty liver disease with chronic kidney disease: a systematic review and meta-analysis. PLoS Med 2014; 11: e1001680.

4. Mantovani A, Zaza G, Byrne CD, Lonardo A, Zoppini G, Bonora E, Targher G. Nonalcoholic fatty liver disease increases risk of incident chronic kidney disease: a systematic review and metaanalysis. Metabolism 2018; 79: 64-76.

5. Glassock RJ, Warnock DG, Delanaye P. The global burden of chronic kidney disease: estimates, variability and pitfalls. Nat Rev Nephrol 2017; 13: 104-114.

6. Mills KT, Xu Y, Zhang W, Bundy JD, Chen CS, Kelly TN, Chen J, He J. A systematic analysis of worldwide population-based data on the global burden of chronic kidney disease in 2010. Kidney Int 2015; 88: 950-957.

7. Go AS, Chertow GM, Fan D, McCulloch CE, Hsu CY. Chronic kidney disease and the risks of death, cardiovascular events, and hospitalization. N Engl J Med 2004; 351: 1296-1305.

8. Anstee $\mathrm{QM}$, Seth D, Day CP. Genetic factors that affect risk of alcoholic and nonalcoholic fatty liver disease. Gastroenterology 2016; 150: 1728-1744.

9. Macaluso FS, Maida M, Petta S. Genetic background in nonalcoholic fatty liver disease: A comprehensive review. World J Gastroenterol 2015; 21: 11088-11111.

10. Musso G, Cassader M, Gambino R. PNPLA3 rs738409 and TM6SF2 rs58542926 gene variants affect renal disease and function in nonalcoholic fatty liver disease. Hepatology 2015; 62: 658659. 
11. Oniki K, Saruwatari J, Izuka T, Kajiwara A, Morita K, Sakata M, Otake K, Ogata Y, Nakagawa K. Influence of the PNPLA3 rs738409 polymorphism on non-alcoholic fatty liver disease and renal function among normal weight subjects. PLoS One 2015; 10: e0132640.

12. Mantovani A, Sani E, Fassio A, Colecchia A, Viapiana O, Gatti D, Idolazzi L, Rossini M, Salvagno G, Lippi G, Zoppini G, Byrne CD, Bonora E, Targher G. Association between non-alcoholic fatty liver disease and bone turnover biomarkers in post-menopausal women with type 2 diabetes. Diabetes Metab 2018 Oct 10. pii: S1262-3636(18)30195-2. doi: 10.1016/j.diabet.2018.10.001 [Epub ahead of print].

13. Levey AS, Stevens LA, Schmid CH, Zhang YL, Castro AF, Feldman HI, Kusek JW, Eggers P, Van Lente F, Greene T, Coresh J; CKD-EPI (Chronic Kidney Disease Epidemiology Collaboration). A new equation to estimate glomerular filtration rate. Ann Intern Med 2009; 150: 604-612.

14. Levey AS, Bosch JP, Lewis JB, Greene T, Rogers N, Roth D. Modification of Diet in Renal Disease Study Group. A more accurate method to estimate glomerular filtration rate from serum creatinine: a new prediction equation. Ann Intern Med 1999; 130: 461-470.

15. Targher G, Zoppini G, Mantovani W, Chonchol M, Negri C, Stoico V, Mantovani A, De Santi F, Bonora E. Comparison of two creatinine-based estimating equations in predicting all-cause and cardiovascular mortality in patients with type 2 diabetes. Diabetes Care 2012; 35: 2347-2353.

16. Byrne CD, Patel J, Scorletti E, Targher G. Tests for diagnosing and monitoring non-alcoholic fatty liver disease in adults. BMJ 2018; 362: k2734.

17. Kozakova M, Palombo C, Eng MP, Dekker J, Flyvbjerg A, Mitrakou A, Gastaldelli A, Ferrannini E; RISC Investigators. Fatty liver index, gamma-glutamyltransferase, and early carotid plaques. Hepatology 2012; 55: 1406-1415.

18. Lee YH, Jung KS, Kim SU, Yoon HJ, Yun YJ, Lee BW, Kang ES, Han KH, Lee HC, Cha BS. Sarcopaenia is associated with NAFLD independently of obesity and insulin resistance: Nationwide surveys (KNHANES 2008-2011). J Hepatol 2015; 63: 486-493. 
19. Bedogni G, Bellentani S, Miglioli L, Masutti F, Passalacqua M, Castiglione A, Tiribelli C. The Fatty Liver Index: a simple and accurate predictor of hepatic steatosis in the general population. BMC Gastroenterol 2006; 6: 33.

20. Hernaez R, Lazo M, Bonekamp S, Kamel I, Brancati FL, Guallar E, Clark JM. Diagnostic accuracy and reliability of ultrasonography for the detection of fatty liver: a meta-analysis. Hepatology 2011; 54: 1082-1090.

21. Guiu B, Crevisy-Girod E, Binquet C, Duvillard L, Masson D, Lepage C, Hamza S, Krausé D, Verges B, Minello A, Cercueil JP, Hillon P, Petit JM. Prediction for steatosis in type-2 diabetes: clinicobiological markers versus ${ }^{1} \mathrm{H}-\mathrm{MR}$ spectroscopy. Eur Radiol 2012; 22: 855-863.

22. Cuthbertson DJ, Weickert MO, Lythgoe D, Sprung VS, Dobson R, Shoajee-Moradie F, Umpleby M, Pfeiffer AF, Thomas EL, Bell JD, Jones H, Kemp GJ. External validation of the fatty liver index and lipid accumulation product indices, using ${ }^{1} \mathrm{H}$-magnetic resonance spectroscopy, to identify hepatic steatosis in healthy controls and obese, insulin-resistant individuals. Eur J Endocrinol 2014; 171: 561-569.

23. McPherson S, Hardy T, Dufour JF, Petta S, Romero-Gomez M, Allison M, Oliveira CP, Francque S, Van Gaal L, Schattenberg JM, Tiniakos D, Burt A, Bugianesi E, Ratziu V, Day CP, Anstee QM. Age as a confounding factor for the accurate non-invasive diagnosis of advanced NAFLD fibrosis. Am J Gastroenterol 2017; 112: 740-751.

24. Romeo S, Kozlitina J, Xing C, Pertsemlidis A, Cox D, Pennacchio LA, Boerwinkle E, Cohen JC, Hobbs HH. Genetic variation in PNPLA3 confers susceptibility to nonalcoholic fatty liver disease. Nat Genet 2008; 40: 1461-1465.

25. Donati B, Motta BM, Pingitore P, Meroni M, Pietrelli A, Alisi A, Petta S, Xing C6, Dongiovanni P, del Menico B, Rametta R, Mancina RM2, Badiali S, Fracanzani AL1, Craxì A, Fargion S, Nobili V, Romeo S, Valenti L. The rs2294918 E434K variant modulates patatin-like phospholipase domaincontaining 3 expression and liver damage. Hepatology 2016; 63: 787-798. 
26. Mitsche MA, Hobbs HH, Cohen JC. Patatin-like phospholipase domain-containing protein 3 promotes transfer of essential fatty acids from triglycerides to phospholipids in hepatic lipid droplets. J Biol Chem 2018; 293: 6958-6968.

27. Seko Y, Yamaguchi K, Itoh Y. The genetic backgrounds in nonalcoholic fatty liver disease Clin J Gastroenterol 2018; 11: 97-102.

28. Pirazzi C, Valenti L, Motta BM, Pingitore P, Hedfalk K, Mancina RM, Burza MA, Indiveri C, Ferro Y, Montalcini T, Maglio C, Dongiovanni P, Fargion S, Rametta R, Pujia A, Andersson L, Ghosal S, Levin M, Wiklund O, lacovino M, Borén J, Romeo S. PNPLA3 has retinyl-palmitate lipase activity in human hepatic stellate cells. Hum Mol Genet 2014; 23: 4077- 4085.

29. Bruschi FV, Claudel T, Tardelli M, Caligiuri A, Stulnig TM, Marra F, Trauner M. The PNPLA3 I148M variant modulates the fibrogenic phenotype of human hepatic stellate cells. Hepatology 2017; 65: 1875-1890.

30. Kramann R, Humphreys BD. Kidney pericytes: roles in regeneration and fibrosis. Semin Nephrol 2014; 34: 374-383.

31. Romeo S, Dongiovanni P, Petta S, Pihalajamaki J, Valenti L. Reply: To PMID 25251399. Hepatology $2015 ; 62: 660$.

32. Duffield JS. Cellular and molecular mechanisms in kidney fibrosis. J Clin Invest 2014; 124: 22992306. 
Table 1. Main clinical and biochemical characteristics of post-menopausal women with noninsulin treated type 2 diabetes, stratified by PNPLA3 rs738409 polymorphism.

\begin{tabular}{|c|c|c|c|c|}
\hline & $\begin{array}{l}\text { CC genotype } \\
(n=52)\end{array}$ & $\begin{array}{l}\text { GC genotype } \\
(n=41)\end{array}$ & $\begin{array}{l}\text { GG genotype } \\
(n=8)\end{array}$ & $P$ value \\
\hline Age (years) & $71.6 \pm 9$ & $71.4 \pm 8$ & $74.2 \pm 8$ & 0.68 \\
\hline Weight (kg) & $74.4 \pm 14$ & $71.9 \pm 11$ & $76.2 \pm 11$ & 0.58 \\
\hline $\mathrm{BMI}\left(\mathrm{kg} / \mathrm{m}^{2}\right)$ & $29.9 \pm 5$ & $30.4 \pm 5$ & $28.9 \pm 5$ & 0.55 \\
\hline Waist circumference $(\mathrm{cm})$ & $100.7 \pm 14$ & $97.0 \pm 11$ & $98.0 \pm 6$ & 0.36 \\
\hline Diabetes duration (years) & $11.7 \pm 9$ & $13.6 \pm 9$ & $10.6 \pm 8$ & 0.51 \\
\hline Current smokers (\%) & 17.3 & 14.6 & 12.5 & 0.32 \\
\hline Systolic blood pressure (mmHg) & $140 \pm 17$ & $138 \pm 17$ & $143 \pm 13$ & 0.68 \\
\hline Diastolic blood pressure ( $\mathrm{mmHg}$ ) & $78 \pm 10$ & $74 \pm 8$ & $77 \pm 8$ & 0.18 \\
\hline White blood count $\left(10^{9} / \mathrm{L}\right)$ & $7.2 \pm 1.3$ & $7.2 \pm 2.3$ & $7.2 \pm 1.1$ & 0.98 \\
\hline Hemoglobin (g/L) & $131.2 \pm 10$ & $131.3 \pm 9$ & $132.2 \pm 12$ & 0.96 \\
\hline Platelet count $\left(10^{9} / \mathrm{L}\right)$ & $251 \pm 62$ & $260 \pm 54$ & $262 \pm 47$ & 0.71 \\
\hline Total cholesterol (mmol/L) & $4.08 \pm 0.8$ & $4.13 \pm 0.9$ & $4.26 \pm 0.6$ & 0.86 \\
\hline LDL cholesterol (mmol/L) & $2.01 \pm 0.6$ & $2.10 \pm 0.8$ & $2.21 \pm 0.5$ & 0.66 \\
\hline HDL cholesterol (mmol/L) & $1.56 \pm 0.4$ & $1.49 \pm 0.3$ & $1.44 \pm 0.3$ & 0.40 \\
\hline Triglycerides (mmol/L) & $1.34 \pm 0.5$ & $1.32 \pm 0.6$ & $1.82 \pm 0.6$ & 0.10 \\
\hline Fasting glucose (mmol/L) & $6.9 \pm 1.1$ & $7.4 \pm 1.9$ & $6.9 \pm 1.1$ & 0.23 \\
\hline Fasting insulin (mIU/L) & $8.9 \pm 6.2$ & $9.3 \pm 9.4$ & $15.0 \pm 7.9$ & 0.12 \\
\hline HOMA-insulin resistance score & $2.3(1.4-3.3)$ & $2.1(1.1-3.7)$ & $4.3(2.7-6.7)$ & 0.32 \\
\hline $\mathrm{HbA} 1 \mathrm{c}(\mathrm{mmol} / \mathrm{mol} \mathrm{Hb})$ & $52.1 \pm 7.7$ & $51.6 \pm 8.9$ & $51.4 \pm 6.2$ & 0.95 \\
\hline C-reactive protein (mg/L) & $2.1(0.8-3.9)$ & $1.1(0.4-3.1)$ & $1.7(0.8-5.1)$ & 0.90 \\
\hline Albumin (g/L) & $44.2 \pm 2.6$ & $44.6 \pm 2.4$ & $44.4 \pm 2.9$ & 0.73 \\
\hline AST (IU/L) & $24 \pm 8$ & $22 \pm 6$ & $28 \pm 6$ & 0.09 \\
\hline ALT (IU/L) & $15 \pm 7$ & $12 \pm 5$ & $16 \pm 9$ & 0.12 \\
\hline GGT (IU/L) & $25 \pm 24$ & $20 \pm 18$ & $29 \pm 18$ & 0.32 \\
\hline Albuminuria (mg/g) & $3.3(2.9-6.6)$ & $3.6(2.8-5.9)$ & $4.9(3.1-28)$ & 0.46 \\
\hline Abnormal albuminuria (\%) & 7.7 & 8.0 & 28.6 & 0.09 \\
\hline CKD (\%) & 13.5 & 24.4 & 50.0 & $=0.04$ \\
\hline Hypertension (\%) & 80.7 & 87.8 & 87.5 & 0.63 \\
\hline Ischemic heart disease (\%) & 11.5 & 12.2 & 12.5 & 0.99 \\
\hline Diabetic retinopathy, any degree (\%) & 3.8 & 9.8 & 12.5 & 0.53 \\
\hline Metformin (\%) & 73.1 & 82.9 & 87.5 & 0.42 \\
\hline Sulfonylureas (\%) & 28.8 & 36.6 & 12.5 & 0.37 \\
\hline Pioglitazone (\%) & 5.8 & 2.4 & 0.0 & 0.60 \\
\hline DPP-4 inhibitors (\%) & 28.8 & 21.9 & 25.0 & 0.75 \\
\hline GLP-1 analogues (\%) & 11.5 & 14.6 & 0.0 & 0.50 \\
\hline SGLT-2 inhibitors (\%) & 5.8 & 7.3 & 0.0 & 0.72 \\
\hline Anti-platelet drugs (\%) & 46.1 & 48.8 & 50.0 & 0.96 \\
\hline Beta-blockers (\%) & 30.8 & 31.5 & 37.5 & 0.93 \\
\hline ARB/ACE-inhibitors (\%) & 63.5 & 73.2 & 75.0 & 0.56 \\
\hline Calcium-channel antagonists (\%) & 19.2 & 19.5 & 12.5 & 0.89 \\
\hline Diuretics (\%) & 25.0 & 48.8 & 37.5 & 0.08 \\
\hline Statins (\%) & 80.8 & 73.2 & 75.0 & 0.68 \\
\hline FIB-4 score >2.67 (\%) & 17.3 & 9.9 & 25.0 & 0.40 \\
\hline $\mathrm{FLI} \geq 60(\%)$ & 46.2 & 39.0 & 62.5 & 0.45 \\
\hline Hepatic steatosis on US (\%), $n=77$ & 85.0 & 73.3 & 71.4 & 0.43 \\
\hline $\begin{array}{l}\text { FLI } \geq 60 \text { and/or hepatic steatosis on } \\
\text { US (\%), } n=77\end{array}$ & 85.0 & 85.7 & 76.7 & 0.64 \\
\hline
\end{tabular}

Sample size, $n=101$, unless where indicated. Data are expressed as means \pm SD, medians or percentages. Differences between the groups were tested by the Fisher's exact test for categorical variables, the one-way ANOVA for normally distributed continuous variables, and the KruskalWallis test for non-normally distributed variables.

CKD was defined as eGFR CKD-EPI $_{100} \mathrm{ml} / \mathrm{min} / 1.73 \mathrm{~m}^{2}$ or abnormal albuminuria (urinary ACR $\geq 30 \mathrm{mg} / \mathrm{g}$ creatinine).

Abbreviations: ACE: angiotensin-converting enzyme; ALT: alanine aminotransferase; ARB: angiotensin II receptor blocker; AST: aspartate aminotransferase; BMI: body mass index; CKD: chronic kidney disease; DPP-4: dipeptidyl peptidase-4; FIB-4, fibrosis-4 score; FLI, fatty liver index; GGT: gamma-glutamyltransferase; GLP-1: glucagon-like peptide-1; HOMA: homeostasis model assessment; SGLT-2: sodium/glucose cotransporter-2; US: ultrasonography. 
Table 2. Multivariable Linear Regression Analyses - association between PNPLA3 rs738409 GG genotype and kidney function (expressed as 1-SD decrement in either eGFR CKD-EPI $_{\text {or }}$ eGFR $\mathrm{R}_{M \mathrm{DD}}$ ) in post-menopausal women with type 2 diabetes.

\begin{tabular}{|c|c|c|c|}
\hline & B coefficient(s) & 95\% Confidence Interval & $P$ value \\
\hline \multicolumn{4}{|c|}{ Adjusted Model 1} \\
\hline \multicolumn{4}{|c|}{ eGFR $_{\text {CKD-EPI }}$ for 1-SD decrement (i.e., $16 \mathrm{ml} / \mathrm{min} / 1.73 \mathrm{~m}^{2}$ ) } \\
\hline \multicolumn{4}{|c|}{ PNPLA3 rs738409 } \\
\hline CC genotype & Ref. & Ref. & - \\
\hline GC genotype & -5.08 & -11.2 to -0.39 & 0.04 \\
\hline GG genotype & -15.2 & -25.4 to -5.07 & $=0.004$ \\
\hline \multicolumn{4}{|c|}{$\mathrm{eGFR}_{\mathrm{MDRD}}$ for 1-SD decrement (i.e., $20 \mathrm{ml} / \mathrm{min} / 1.73 \mathrm{~m}^{2}$ ) } \\
\hline \multicolumn{4}{|c|}{ PNPLA3 rs738409 } \\
\hline CC genotype & Ref. & Ref. & - \\
\hline GC genotype & -6.44 & -14.3 to +1.39 & 0.11 \\
\hline GG genotype & -19.0 & -33.7 to -4.27 & $=0.01$ \\
\hline \multicolumn{4}{|c|}{ Adjusted Model 2} \\
\hline \multicolumn{4}{|c|}{ eGFR $_{\text {CKD-EPI }}$ for 1-SD decrement (i.e., $16 \mathrm{ml} / \mathrm{min} / 1.73 \mathrm{~m}^{2}$ ) } \\
\hline \multicolumn{4}{|c|}{ PNPLA3 rs738409 } \\
\hline CC genotype & Ref. & Ref. & - \\
\hline GC genotype & -5.67 & -11.3 to -0.08 & $=0.05$ \\
\hline GG genotype & -15.5 & -26.0 to -5.04 & $=0.004$ \\
\hline \multicolumn{4}{|c|}{$\mathrm{eGFR}_{\mathrm{MDRD}}$ for 1-SD decrement (i.e., $20 \mathrm{ml} / \mathrm{min} / 1.73 \mathrm{~m}^{2}$ ) } \\
\hline \multicolumn{4}{|c|}{ PNPLA3 rs738409 } \\
\hline CC genotype & Ref. & Ref. & - \\
\hline GC genotype & -5.94 & -14.1 to +2.19 & 0.15 \\
\hline GG genotype & -19.4 & -34.7 to -4.19 & $=0.01$ \\
\hline
\end{tabular}

Sample size, $n=101$. Data are expressed as beta coefficients and $95 \%$ confidence intervals $(\mathrm{Cl})$ as tested by multivariable linear regression analysis.

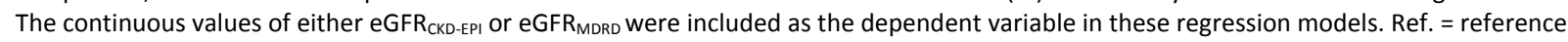
category.

Multivariable linear regression model 1 was adjusted for age, duration of diabetes, HbA1c, systolic blood pressure, HOMA-IR score, use of antihypertensive drugs and presence of NAFLD (as assessed by FLI $\geq 60$ ). Model 2 was adjusted for the same set of covariates included in model 1 plus smoking history, statin use, prior ischemic heart disease and abnormal albuminuria (urinary ACR $\geq 30 \mathrm{mg} / \mathrm{g}$ ). 
Table 3. Logistic Regression Analyses - Association between PNPLA3 rs738409 GG genotype and risk of prevalent CKD in post-menopausal women with type 2 diabetes.

\begin{tabular}{llll}
\hline & Odds Ratio(s) & $\mathbf{9 5 \%}$ Confidence Interval & $\boldsymbol{P}$ value \\
\hline Unadjusted model & & & \\
\hline PNPLA3 rs738409 & & & - \\
CC genotype & Ref. & Ref. & 0.18 \\
GC genotype & 2.07 & $0.71-6.03$ & $=0.02$ \\
GG genotype & 6.43 & $1.29-31.8$ & \\
\hline Adjusted model & & & - \\
\hline PNPLA3 rs738409 & & & $=0.04$ \\
CC genotype & Ref. & Ref. & $=0.03$ \\
GC genotype & 3.97 & $1.02-15.4$ & $1.26-41.4$ \\
GG genotype & 8.05 & & \\
\hline
\end{tabular}

Sample size, $n=101$. Data are expressed as odds ratios $\pm 95 \%$ confidence intervals $(\mathrm{Cl}$ ) as assessed by either univariate (unadjusted) or multivariable logistic regression analyses. Presence of CKD (defined as eGFR CKD-EPI $_{60} \mathrm{ml} / \mathrm{min} / 1.73 \mathrm{~m}^{2}$ or abnormal albuminuria) was included as the dependent variable in these regression models. Ref. = reference category.

All these multivariable regression models were adjusted for age, duration of diabetes, HbA1c, systolic blood pressure, HOMA-IR score, use of antihypertensive drugs and presence of NAFLD (as assessed by FLI $\geq 60$ ). 


\section{FIGURE LEGEND}

Figure 1. Box plot of estimated glomerular filtration rate values [i.e., using the eGFR $\operatorname{CKD}_{\mathrm{CPI}}(\mathrm{A})$ and eGFR $\mathrm{R}_{\mathrm{MDD}}$ equations (B)] of post-menopausal type 2 diabetic women, stratified by PNPLA3 rs738409 polymorphism. P-values for the trend by one-way ANOVA. A post-hoc Tukey's HSD (Honestly Significant Difference) test was used for pairwise comparisons of means among the three groups of patients. The central rectangle spans the $1^{\text {st }}$ quartile to the $3^{\text {rd }}$ quartile (the interquartile range $[\mathrm{IQR}])$. The segment inside the rectangle shows the median and "whiskers" above and below the box show the locations of $1.5 \times$ IQR values. 
Supplementary Table 1. Main clinical, biochemical and PNPLA3 rs738409 genotype of postmenopausal women with non-insulin treated type 2 diabetes, stratified by both CKD (defined as eGFR CKD-EPI $_{1}<0 \mathrm{ml} / \mathrm{min} / 1.73 \mathrm{~m}^{2}$ or abnormal albuminuria) and NAFLD (as detected by FLI $\geq 60$ ).

\begin{tabular}{|c|c|c|c|c|c|}
\hline & $\begin{array}{l}\text { No CKD/No } \\
\text { NAFLD } \\
(n=51) \\
\end{array}$ & $\begin{array}{l}\text { Yes CKD/No } \\
\text { NAFLD } \\
(n=5)\end{array}$ & $\begin{array}{l}\text { No CKD/Yes } \\
\text { NAFLD } \\
(n=29)\end{array}$ & $\begin{array}{l}\text { Yes CKD/Yes } \\
\text { NAFLD } \\
(n=16)\end{array}$ & $P$ value \\
\hline Age (years) & $72.6 \pm 7.9$ & $75.0 \pm 6.2$ & $68.6 \pm 9.2$ & $73.6 \pm 9.1$ & 0.12 \\
\hline BMI $\left(\mathrm{kg} / \mathrm{m}^{2}\right)$ & $26.5 \pm 3.0$ & $26.7 \pm 4.4$ & $33.5 \pm 4.8$ & $32.6 \pm 3.7$ & $<0.001$ \\
\hline Waist circumference $(\mathrm{cm})$ & $91.7 \pm 7.7$ & $92.2 \pm 9.7$ & $107.5 \pm 9.7$ & $109.1 \pm 14.9$ & $<0.001$ \\
\hline Diabetes duration (years) & $11.4 \pm 7.9$ & $12.0 \pm 10.4$ & $12.4 \pm 9.9$ & $15.6 \pm 7.4$ & 0.44 \\
\hline Current smokers (\%) & 11.8 & 40.0 & 17.2 & 12.5 & 0.75 \\
\hline Systolic blood pressure (mmHg) & $138 \pm 16$ & $143 \pm 23$ & $139 \pm 20$ & $144 \pm 10$ & 0.52 \\
\hline Diastolic blood pressure (mmHg) & $76 \pm 7$ & $80 \pm 14$ & $77 \pm 11$ & $75 \pm 9$ & 0.56 \\
\hline Total cholesterol (mg/dL) & $155.7 \pm 30$ & $149.8 \pm 53$ & $168.7 \pm 32$ & $163.0 \pm 32$ & 0.31 \\
\hline HDL cholesterol (mg/dL) & $63.2 \pm 13$ & $56.8 \pm 17$ & $54.7 \pm 12$ & $55.9 \pm 13$ & 0.02 \\
\hline Triglycerides (mg/dL) & $93.9 \pm 32$ & $121.0 \pm 64$ & $148.7 \pm 54$ & $153.5 \pm 65$ & $<0.001$ \\
\hline Fasting glucose (mmol/L) & $6.8 \pm 1.3$ & $6.5 \pm 0.7$ & $7.6 \pm 1.7$ & $7.6 \pm 1.6$ & 0.03 \\
\hline Fasting insulin (mIU/L) & $6.6 \pm 4.7$ & $6.8 \pm 4.3$ & $12.1 \pm 7.5$ & $14.9 \pm 11.3$ & $<0.001$ \\
\hline HOMA-insulin resistance score & $1.5(1.0-2.5)$ & $2.0(0.7-3.3)$ & $3.0(2.1-6.2)$ & $3.9(2.3-6.6)$ & $<0.001$ \\
\hline AST (IU/L) & $23 \pm 8$ & $24 \pm 5$ & $24 \pm 8$ & $24 \pm 6$ & 0.83 \\
\hline ALT (IU/L) & $12 \pm 4$ & $13 \pm 6$ & $16 \pm 8$ & $13 \pm 4$ & 0.12 \\
\hline GGT (IU/L) & $18 \pm 11$ & $10 \pm 3$ & $30 \pm 20$ & $29 \pm 16$ & 0.02 \\
\hline PNPLA3 genotype (\%) & & & & & 0.09 \\
\hline $\mathrm{CC}$ & 51.0 & 40.0 & 65.5 & 31.2 & \\
\hline GC & 43.1 & 60.0 & 31.0 & 43.8 & \\
\hline GG & 5.9 & 0 & 3.5 & 25.0 & \\
\hline
\end{tabular}

Sample size, $n=101$. Data are expressed as means \pm SD, medians or percentages. Differences between the groups were tested by the Fisher's exact test for categorical variables, the one-way ANOVA for normally distributed continuous variables, and the Kruskal-Wallis test for non-normally distributed variables.

Abbreviations: ALT: alanine aminotransferase; AST: aspartate aminotransferase; BMI: body mass index; GGT: gammaglutamyltransferase; HOMA: homeostasis model assessment. 
Supplementary Table 2. Multivariable Linear Regression Analyses - Association between PNPLA3 rs738409 GG genotype and kidney function (expressed as 1-SD decrement in either eGFR CKD-EPI $_{\text {Or }}$ eGFR $R_{M D R D}$ ) in the subgroup of post-menopausal T2DM women with available liver ultrasonography.

\begin{tabular}{|c|c|c|c|}
\hline & B coefficient(s) & 95\% Confidence Interval & $P$ value \\
\hline \multicolumn{4}{|c|}{ Adjusted Model 1} \\
\hline \multicolumn{4}{|c|}{$\mathrm{eGFR}_{\mathrm{CKD}-\mathrm{EPI}}$ for 1-SD decrement (i.e, $16 \mathrm{ml} / \mathrm{min} / 1.73 \mathrm{~m}^{2}$ ) } \\
\hline \multicolumn{4}{|l|}{ PNPLA3 rs738409 } \\
\hline CC genotype & Ref. & Ref. & - \\
\hline GC genotype & -4.81 & -10.9 to +1.30 & 0.12 \\
\hline GG genotype & -14.8 & -25.2 to -2.90 & $=0.01$ \\
\hline \multicolumn{4}{|c|}{$\mathrm{eGFR}_{\mathrm{MDRD}}$ for 1-SD decrement (i.e, $20 \mathrm{ml} / \mathrm{min} / 1.73 \mathrm{~m}^{2}$ ) } \\
\hline \multicolumn{4}{|c|}{ PNPLA3 rs738409 } \\
\hline CC genotype & Ref. & Ref. & - \\
\hline GC genotype & -5.13 & -14.4 to +4.09 & 0.27 \\
\hline GG genotype & -17.9 & -34.9 to -1.11 & $=0.04$ \\
\hline \multicolumn{4}{|l|}{ Adjusted Model 2} \\
\hline \multicolumn{4}{|c|}{ eGFR $_{\text {CKD-EPI }}$ for $1-S D$ decrement (i.e., $16 \mathrm{ml} / \mathrm{min} / 1.73 \mathrm{~m}^{2}$ ) } \\
\hline \multicolumn{4}{|c|}{ PNPLA3 rs738409 } \\
\hline CC genotype & Ref. & Ref. & - \\
\hline GC genotype & -4.72 & -11.2 to +1.75 & 0.15 \\
\hline GG genotype & -14.1 & -25.9 to -2.39 & $=0.02$ \\
\hline \multicolumn{4}{|c|}{ eGFR $_{\text {MDRD }}$ for 1-SD decrement (i.e., $20 \mathrm{ml} / \mathrm{min} / 1.73 \mathrm{~m}^{2}$ ) } \\
\hline \multicolumn{4}{|c|}{ PNPLA3 rs738409 } \\
\hline CC genotype & Ref. & Ref. & - \\
\hline GC genotype & -5.1 & -14.9 to +4.68 & 0.30 \\
\hline GG genotype & -17.1 & -34.9 to -0.69 & $=0.05$ \\
\hline
\end{tabular}

Sample size, $n=77$. Data are expressed as beta coefficients and $95 \%$ confidence intervals (CI) as tested by multivariable linear regression analysis. The continuous values of either eGFR $\mathrm{CKD}_{\mathrm{CPI}}$ or $\mathrm{GGFR}_{\mathrm{MDRD}}$ were included as the dependent variable in these regression models. Ref. $=$ reference category.

Multivariable linear regression model 1 was adjusted for age, duration of diabetes, HbA1c, systolic blood pressure, HOMA-IR score, use of antihypertensive drugs and presence of NAFLD (as detected by ultrasonography). Model 2 was adjusted for the same set of covariates included in model 1 plus smoking, statin use, prior ischemic heart disease and abnormal albuminuria (urinary ACR $\geq 30 \mathrm{mg} / \mathrm{g}$ ). 
Supplementary Table 3. Logistic Regression Analyses - Association between PNPLA3 rs738409 GG genotype and risk of prevalent CKD in the subgroup of post-menopausal T2DM women with available liver ultrasonography.

\begin{tabular}{llll}
\hline & Odds Ratio(s) & $\mathbf{9 5 \%}$ Confidence Interval & $\boldsymbol{P}$ value \\
\hline Unadjusted model & & & \\
\hline PNPLA3 rs738409 & & & - \\
CC genotype & Ref. & Ref. & 0.92 \\
GC genotype & 1.08 & $0.26-4.40$ & $=0.01$ \\
GG genotype & 9.33 & $1.60-54.6$ & - \\
\hline Adjusted model & & & \\
\hline PNPLA3 rs738409 & & & 0.65 \\
CC genotype & Ref. & Ref. & $=0.03$ \\
GC genotype & 1.44 & $0.29-6.93$ & $1.25-62.0$ \\
GG genotype & 8.81 & & \\
\hline
\end{tabular}

Sample size, $n=77$. Data are expressed as odds ratios $\pm 95 \%$ confidence intervals $(\mathrm{Cl})$ as assessed by multivariable logistic regression analysis. The presence of CKD (defined as eGFR $\mathrm{CKD}_{\text {-EPI }}<60 \mathrm{ml} / \mathrm{min} / 1.73 \mathrm{~m}^{2}$ or abnormal albuminuria) was included as the dependent variable of this regression model. Ref. = reference category.

The multivariable regression model was adjusted for age, duration of diabetes, HbA1c, systolic blood pressure, HOMA-IR score, use of antihypertensive drugs and presence of NAFLD (as detected by ultrasonography). 